PAPER

\section{Empirical mode decomposition for self-mixing Doppler signals of hemodynamic optical probes}

To cite this article: Tânia Pereira et al 2013 Physiol. Meas. 34377

View the article online for updates and enhancements.

\section{Related content}

Arterial pulse shape measurement using
$\frac{\text { self-mixing effect in a diode laser }}{\text { J Hast, Risto Myllylä, H Sorvoja et al. }}$
- Non-invasive measurement of local PP by
$\frac{\text { PWUM }}{\text { J Vappou, J Luo, K Okajima et al. }}$
- Single-source PPG-based local pulse
$\frac{\text { wave velocity measurement: a potential }}{\text { cuffless blood pressure estimation }}$
technique
P M Nabeel, J Jayaraj and S
Mohanasankar

\section{Recent citations}

- Analysis of Laser Speckle Contrast
$\frac{\text { Images Variability Using a Novel Empirical }}{\text { Mode Decomposition: Comparison of }}$
$\frac{\text { Results With Laser Doppler Flowmetry }}{\text { Signals Variability }}$
Anne Humeau-Heurtier et al
- Novel Methods for Pulse Wave Velocity
$\frac{\text { Measurement }}{\text { Tânia Pereira et al }}$
- Signal (Stream) synchronization with
$\frac{\text { White noise sources, in biomedical }}{\text { applications }}$
P. Vaz et al




\title{
Empirical mode decomposition for self-mixing Doppler signals of hemodynamic optical probes
}

\author{
Tânia Pereira ${ }^{1,3}$, Pedro Vaz ${ }^{1}$, Tatiana Oliveira ${ }^{1}$, Inês Santos ${ }^{1}$, \\ Helena C Pereira $^{1,2}$, Vânia Almeida ${ }^{1}$, Carlos Correia ${ }^{1}$ \\ and João Cardoso ${ }^{1}$ \\ ${ }^{1}$ Instrumentation Centre, Physics Department, University of Coimbra, Portugal \\ 2 ISA-Intelligent Sensing Anywhere, Coimbra, Portugal \\ E-mail: taniapereira@lei.fis.uc.pt
}

Received 4 September 2012, accepted for publication 30 January 2013

Published 26 February 2013

Online at stacks.iop.org/PM/34/377

\begin{abstract}
A new type of optical probe based on laser Doppler self-mixing technology, for a truly non-contact measurement in a single location, and extraction of the temporal features of the distension wave in the arterial wall, was developed. The monitoring of temporal features allows the assessment of cardiovascular function when measurement is carried out at the carotid artery. An algorithm based on the short-time Fourier transform and empirical mode decomposition was applied to the test setup self-mixing signals for the determination of waveform features, with an accuracy of a few milliseconds and a root mean square error less than $3 \mathrm{~ms}$. In vivo testing signals show great consistency in the measured pulse pressure waveform.
\end{abstract}

Keywords: self-mixing Doppler signals, empirical mode decomposition, hemodynamic parameters

(Some figures may appear in colour only in the online journal)

\section{Introduction}

In a context where cardiovascular diseases are a rising problem in developed countries, there is an increasing need for solutions that monitor vital signals and give an earlier and accurate diagnosis of potential disorders.

An increase in the stiffness of the arterial wall appears to be a common pathological pathway for many factors that lead to initiation and progression of the vascular changes associated with cardiovascular disease (Selzer et al 2001). Functional disturbances of the

3 Author to whom any correspondence should be addressed. 
vascular wall may occur early in the atherosclerotic process, even before the anatomical changes of the thickening of the arterial wall become perceptible. Non-invasive measurement of arterial wall stiffness could become important to identify individuals at risk of cardiovascular disease (Selzer et al 2001).

Local pulse pressure, pulse wave velocity (PWV) and certain waveform characteristics constitute some of the most important cardiovascular risk indicators. In the case of arterial stiffening, which affects artery compliance and therefore the distension profile, the measurement and analysis of the distension waveforms permits the determination of the risk of cardiovascular diseases (Avolio et al 2010).

Central blood pressure waveforms contain specific features related to the cardiac and arterial functions. Information on the interaction between the blood ejection of the left ventricle and the physical properties of the arteries can be determined by the descriptive and quantitative analysis of the arterial pulse pressure waveform (PPW). The main feature points of the PPW are: the systolic peak (SP), generated by ventricle contraction, and the reflection point (RP), originated by reflections of the pressure wave from peripheral sites and dichrotic notch which corresponds to the aortic valve closure. Through pulse wave analysis (PWA), it is possible to compute the temporal occurrence of those features which allow the assessment of important aspects of the cardiovascular function (Avolio et al 2010).

Several studies show that PWA and PWV can be predictive of coronary artery disease (Weber et al 2004) and hypertension, which are related to an increase in arterial stiffness (high PWV), and hypercholesterolemia, that is associated with a reduced aortic stiffness (low PWV) (Dart and Kingwell 2001). The information extracted from the arterial pulse pressure provides insights into the diagnosis and management of many disease states, including diabetes (Korpas et al 2009), diastolic dysfunction (Nelson et al 2010), obstructive sleep apnea (Phillips et al 2005) or rheumatoid arthritis (Klocke et al 2003).

Over the last few decades, numerous conventional non-invasive techniques have been developed to detect cardiovascular pulsation, blood pressure and PPW. The first techniques included the oscillometric methods, stethoscopes, phonocardiograms or manual palpation of superficial arteries (Scalise and Morbiducci 2008), which were inaccurate and lacked sensitivity.

The optical systems based on light emission and backscattered light detection are an alternative that has been outperforming the inherent limitations of other types of systems, in particular due to their high accuracy, high resolution and non-contact skin approach (Pereira et al 2011). The carotid artery seems to be a preferential site for analyzing the wall's thickness since it is an elastic artery and is located relatively close to the heart. The total thickness of the arterial wall is essentially related to the thickness of the intimal layer (Selzer et al 2001, Boutouyrie et al 1995).

Laser Doppler technology is based on the theory of self-mixing interference between the emitted and reflected waves in a moving target, i.e. different wavelengths. This approach simplifies the optical scheme of such devices, as they involve only one optical axis, in opposition to alternative multi-axis schemes using several optical components.

This laser-based technique has been applied in biomedical areas due to its advantageous metrological characteristics with potential to be an alternative to current investigational methodologies. The self-mixing effect was used in hemodynamic applications for PPW analysis in several works (Scalise and Morbiducci 2008, Campo et al 2011, Hast et al 2002, Hong and Fox 1994) but their clinical applications and commercial development are still in the emergent stage. The construction of a new device, using simple and low cost optical and electronic components, with signal processing based on a different algorithm that improves 
the accuracy in the extraction of parameters, will fill the existent gaps in the assessment of the arterial pulse pressure.

The information of the self-mixing signals is mostly encoded in the frequency domain (Figuerias 2012) and thus, time-frequency signal processing techniques are necessary to extract important data. The commonly used technique to process self-mixing interferometric signals is the Fourier transform (FT) that allows both magnitude and phase analysis (Hast et al 2002, Guo and Wang 2007, Wang 2001, Scalise and Paone 2002). The Doppler spectrograms from the FT of Doppler signals permit the extraction of the movement profile and the absolute displacement. However, in this application, for the hemodynamic parameter extraction, the main issue is the identification of the specific features in the Doppler signals that correspond to inflection points in the PPW. As a result, an effective and robust algorithm is necessary for the detection of the minimum points in the Doppler signal after the application of the FT.

The empirical mode decomposition (EMD) has been used to process non-stationary signals (Junsheng et al 2006) and was applied in distinct fields such as spacecraft signal analysis (Hai et al 2008) and face recognition (Gallego-Jutglà and Solé-Casals 2012). This study aims to apply these methods to self-mixing signals to improve the accuracy in the detection of the local minimum points of the Doppler spectrograms.

In this work, an optical probe based on laser Doppler technology was developed, which uses self-mixing signals to obtain the PPW measured in the carotid, and determines the temporal occurrence of the feature wave points. To achieve these goals, a short-time Fourier transform (STFT)-based algorithm and EMD were applied to the self-mixing signals, as explained below.

\section{Instrumentation}

\subsection{Optical probe}

The use of laser diodes (LD), which are simultaneously laser-emitting and photo-detectors, is based on the theory of the self-mixing interference between the emitted wave and the resulting Doppler shifted wave.

The Doppler effect is characterized by a change in the frequency of the received wave, reflected from a moving structure, when related to the wave frequency of the emitter. The variation in the received frequency $\left(f^{\prime}\right)$ is related to the velocity of the source, the velocity of the moving structure and the frequency of the emitted wave according to

$$
f^{\prime}=f \frac{v \pm v_{r}}{v+v_{s}}
$$

In equation (1), $f^{\prime}$ represents the Doppler frequency determined by means of the original frequency, $f$, the wave velocity in a specific medium, $v$, the velocity of the reflector, $v_{r}$, and the velocity of the source, $v_{s}$.

The principle of self-mixing interferometry is based on the optical feedback that occurs when the light beam is reflected by a moving target and is directly coupled back into the laserdiode active cavity. The interference of the back-coupled light with the original light, in the cavity, causes variations in both amplitude and frequency of the optical power emitted from the laser diode. These variations are detected as a current signal with a photodiode accommodated in the laser-diode package (Giuliani et al 2002). In the laser cavity, the original light mixes with the Doppler-frequency shifted light and originates a beat wave. The resulting frequency shift is related with the vibration velocity of the moving target, i.e. of the carotid membrane. Theoretically, the velocity of the skin movements is proportional to the time derivative of the blood pressure profile within the underlying vasculature (Hast et al 2002). 


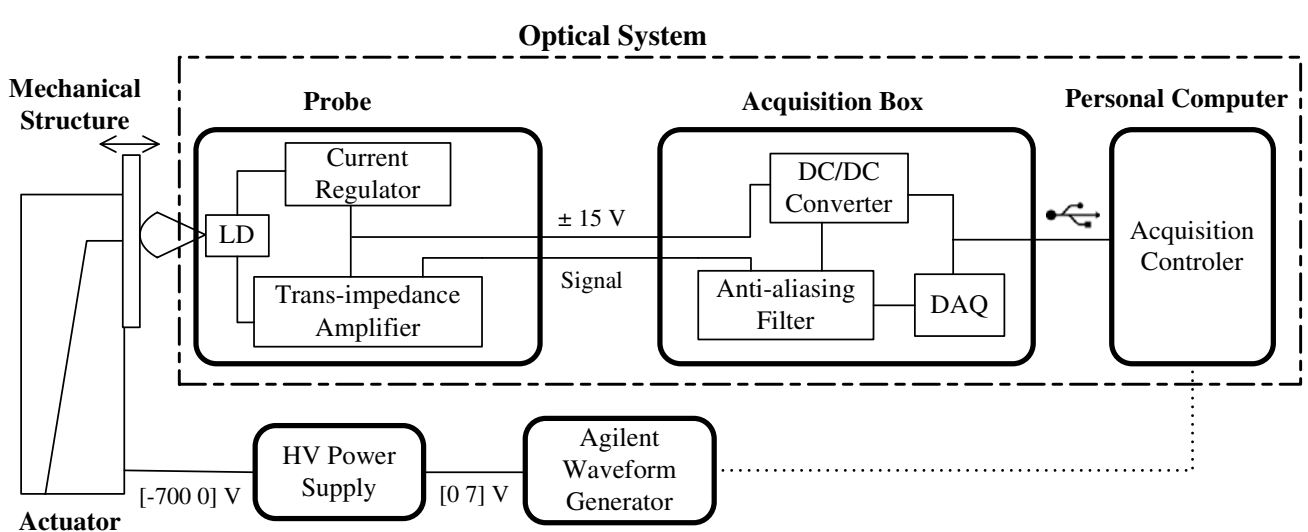

Figure 1. Block diagram of the optical system and all its minor components and simple diagram of the implemented test setup.

A laser diode from Laser Components (Visible Laser Diode ADL-65075TL) with a peak wavelength of $635 \mathrm{~nm}$ and $5 \mathrm{~mW}$ output power is used. The relevant regulations according to ICNIRP Guidelines (ICNIRP 2000) and the applicable accident prevention regulations were taken into account.

The developed probe was mounted perpendicularly to a printed circuit board layer. The probe includes a laser diode driven by a constant current as well as signal conditioning electronics based on a trans-impedance amplifier (figure 1-probe). The general negative feedback arrangement can be implemented by a voltage regulator; in this case an LM337 is used to drive the laser diode. It keeps up a constant voltage drop $(1.25 \mathrm{~V})$ across a constant resistor (42 $\Omega$ ); so, a constant current (30 mA) flows through the resistor and load. The electronic amplification circuit is composed of an operational amplifier (Texas Instruments, OPA129UE4) which is an ultra-low bias current monolithic operational amplifier, for a current to voltage conversion (trans-impedance amplifier).

The probe is cased in an ergonomic box with a hole for the laser diode in the front face which permits the emission and reception of light. The developed probe has an ergonomic design to minimize the forces applied and improve the procedure of positioning the probe in the measuring site. The probe should be shaped and textured to be comfortable for both the user and patient. The complete design should be easy to stow and transportable in a clinical setting, compact and agile system.

\subsection{Optical system}

The developed system is composed of an optical probe, a data acquisition box and a personal computer for signal processing (figure 1 -optical system). The data acquisition box comprises an anti-aliasing filter, the data acquisition system (DAQ), a dc/dc converter and a USB connection for primary power supply and interface with the acquisition controller. The $\mathrm{dc} / \mathrm{dc}$ circuit converts the standard USB $+5 \mathrm{~V}$ voltage to the voltages required by the analog electronics $( \pm 15 \mathrm{~V})$ of the optical probe.

A combination of one amplifier (Texas Instruments, TL081), resistors and capacitors is used to implement a single pole active filter. The active filter offers the advantage of providing 'isolation' between stages. This is possible by taking advantage of the high input impedance 
and low output impedance of the operational amplifier. The filters have a unit dc gain of -1 with an attenuation of $-3 \mathrm{~dB}$ at $50 \mathrm{kHz}$ (Mya et al 2009).

The signals were acquired using a 16-bit resolution data acquisition system (National Instruments, USB-6343) and stored for offline analysis using Mathworks Matlab ${ }^{\circledR}$.

\subsection{Test setup}

The test setup, schematically represented in figure 1, was designed to evaluate if the optical probe was able to measure distensions of the same order of magnitude as the ones observed at the skin over the carotid site. The test waveforms were generated by an Agilent 33220A arbitrary waveform generator, which feeds an actuator (700 $\mu \mathrm{m}$ Physik Instrumente GmbH, P-287), biased by a high voltage linear power driver (Physik Instrumente GmbH, E-508). The actuator moves a mechanical structure (MS) as shown in figure 1, lined up with a silicone membrane that reflects the light emitted by the laser diode, similarly to human skin. Each silicone layer was $1 \mathrm{~mm}$ thick and the tests were conducted using four layers $(4 \mathrm{~mm})$. The cardiac-like waveforms, generated in the test setup are similar to healthy individual cardiac waveforms. The actuator reproduces these waveforms with a maximum displacement of $500 \mu \mathrm{m}$.

The optical probe was positioned in front of the MS and detected variations of the reflected light. The signals were acquired through the acquisition box and stored in a personal computer. All algorithms for PWA were developed using Matlab ${ }^{\circledR}$ software, version 7.8.0 (R2009a).

\section{Signal processing}

\subsection{Doppler signal}

All the signals were acquired in the test setup described before. Self-mixing signals were acquired in a long range of sampling frequencies (from $20 \mathrm{kHz}$ up to $200 \mathrm{kHz}$ ) (Hast et al 2002, Guo and Wang 2006, 2007, Wang 2004). The sampling frequency set must ensure that it is always more than twice the Doppler frequency to be measured with this system. In the arterial pulse pressure, the velocity expected for the carotid movement is approximately $4500 \mu \mathrm{m} \mathrm{s}^{-1}$ (van Ittersum et al 2004), that corresponds to a maximum Doppler frequency of $20 \mathrm{kHz}$. A $100 \mathrm{kHz}$ sampling frequency was elected for the acquisition of the signals since it ensures the best compromise between signal quality and computational effort, according to a shape waveform root mean square (RMS) error test. As mentioned above, the self-mixing signals are a non-stationary type of data due to their high dependence on the Doppler effect (Meigas et al 2001). The typical signals acquired in the test setup are shown in figure 2 and the solid line represents the actuator displacement.

\subsection{Algorithm}

The Doppler signals require a time-frequency analysis to extract most of the physiologically important parameters. An algorithm based on the STFT and EMD was used to find the feature points of the pulse waveform as schematically described in figure 3 .

To find the time of occurrence of the clinically relevant feature points of the PPW a STFT was performed. This algorithm was used with a 124-point length Hanning window and a 100point segment overlap for high temporal resolution (Meigas et al 1998). The Fourier length was 1024 points using zero padding of the analyzed signal to obtain a better frequency discretization and the resulting spectrogram is shown in figure 4(a). After these processing stages, a 

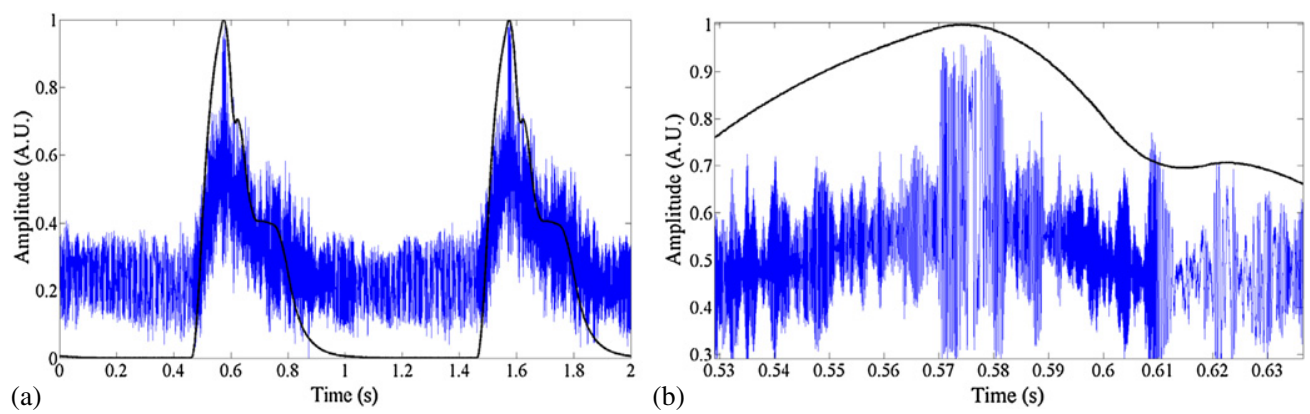

Figure 2. Test setup signals. (a) Self-mixed laser signal acquired in the test setup and the waveform described by the actuator marked by a solid line. (b) Detail of the signals, self-mixing effect.

two-dimensional signal was constructed for a good visualization of the time-frequency spectrum (figure 4(b)).

The STFT provides a two-dimensional matrix with the coefficients representative of the frequencies present in each time window (figure 4(a)). The next step of this algorithm is to find the predominant frequency (figure 4(b)). Numerically, this is achieved by finding the maximum value in every column and representing its values on a time scale, i.e. on a one-dimensional signal. Since the dc value of the signal is extremely high, the zero frequency is the predominant one at every window. However, as the zero frequency does not bear significant information, it was excluded from this search of frequencies in order to find the correct Doppler shift frequency using this algorithm. In addition, a threshold was defined to prevent the determination of image artifacts as predominant frequencies. Thereafter, a Doppler shift frequency was reconstructed along time from the original self-mixing signal. The reconstructed signal represents the most predominant frequency of the original self-mixing signal over time, which corresponds to the Doppler frequency shift.

Figure 4(b) shows the reconstructed signal which has strong correlation with the derivative of the signal reproduced by the actuator, despite the presence of high noise level. To remove the noise present in the reconstructed signal an EMD-based algorithm was conducted. This method intended to create a multiscale decomposition and a time-frequency analysis of the reconstructed signal (Gallego-Jutglà and Solé-Casals 2012).

The PPW undergoes great variability in the shape and duration between individuals; for these reasons a self-adaptive algorithm represents an advantage in this analysis. The computation of the EMD does not require any previously known value of the signal, which accounts to be an eligible tool for that purpose.

The EMD and wavelet-transform, which represent standard techniques, provide the decomposition of a signal into different time scales. The main difference is that the EMD performs the signal decomposition adaptively and based exclusively on the available timeseries, whereas the wavelet-transform uses a set of pre-fixed filters based on the choice of a mother wavelet function and scale (Andrade et al 2006).

The arterial pulse pressure signals are the result of several biological processes (cardiac activity and arterial function) that correspond to different time scales in the analyzed signal. According to the EMD procedures, data are decomposed into several fundamental components (decomposition layers), each with a distinct time scale, sequential extraction of energy associated with various intrinsic time scales of the signal starting from finer temporal scales (high frequency modes) to coarser ones (low frequency modes). The iterative process explores 


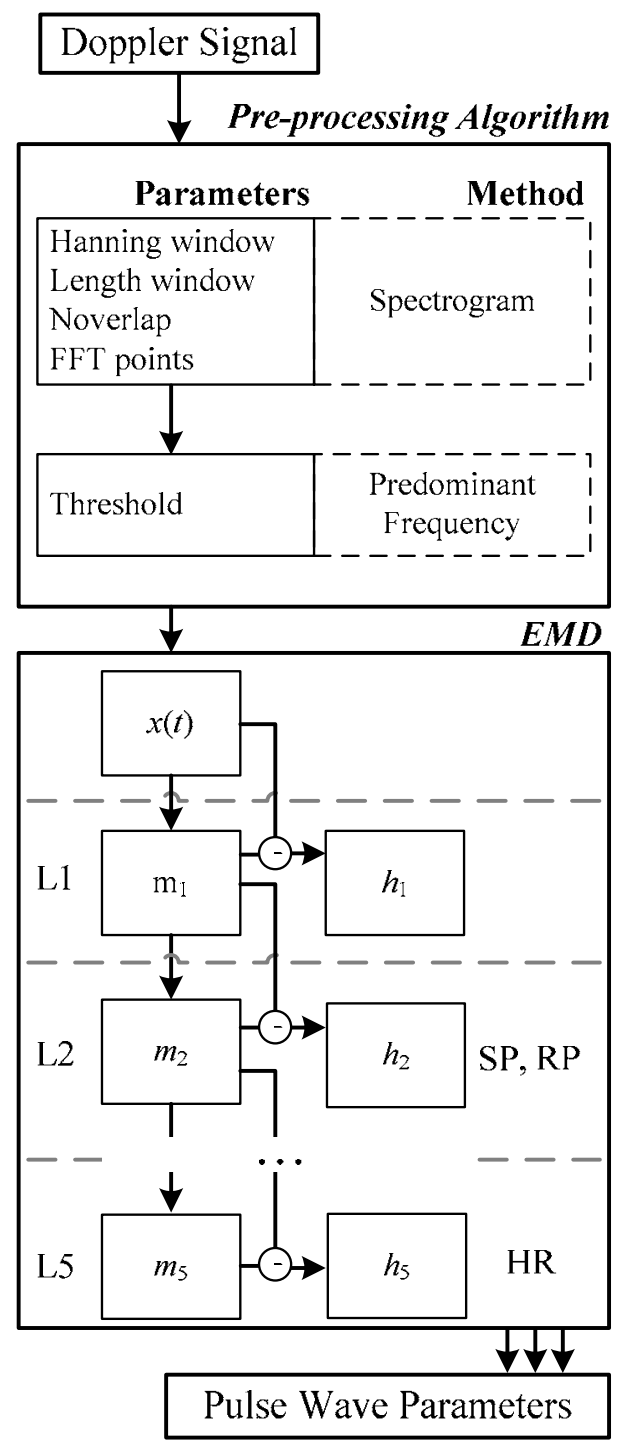

Figure 3. Schematic overview of the algorithm developed. Algorithm for pre-processing the Doppler signals and several levels of the EMD.

sequentially the natural constitutive scales of the signal (Qing 2010), giving an almost direct insight into the physiological mechanisms undergoing in the arterial propagation process.

The applied algorithm is an iterative computation where the upper and lower wave envelopes of the signal are computed using, in this case, a cubic interpolation. Then the mean value of the upper and lower envelopes $(m)$ is subtracted from the original signal $(x)$ yielding $h$, expressed in equation (2). In this case, the main goal is to find the feature points of the pulse waveform, the residue $(h)$ is negligible and the envelope $(m)$ is considered a new signal to decompose. Once the highest frequency is removed from a signal, the same procedure is applied on the residue signal to identify the next highest frequency. The stop 

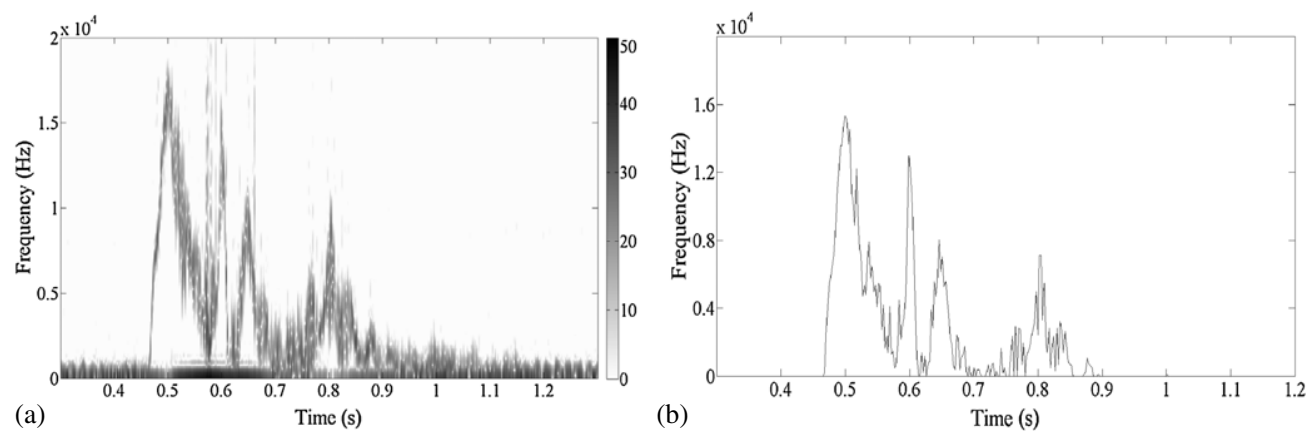

Figure 4. Spectrogram of the original signal. (a) Spectrogram in a conventional gray scale representing the top $50 \mathrm{~dB}$ of the signal. (b) Wave reconstructed from the image spectrogram.

condition implies that the number of zero crossings of $m$ is equal, or differs by 1 , to the number of the extremes (Gallego-Jutglà and Solé-Casals 2012); otherwise it considers $m$ as $x$ and repeats the envelope procedure, equation (3).

$$
\begin{aligned}
& h_{n}(t)=x(t)-m_{n}(t), \\
& x(t)=m_{n}(t) .
\end{aligned}
$$

After the decomposition, for each level two data sets were obtained. The smooth effect was obtained from the envelope of higher levels, so $m$ data represents the filtered signal. The extracted data appeared on the $m$ signal. Due to the cubic interpolation, the decomposed signal's boundaries tend to have very large absolute values which are characteristic of the cubic functions. To solve this problem, the edges of the processed signal were removed without constituting a major problem since the processed signal $(10 \mathrm{~s})$ contains several periods of the original movement. Figure 5 represents the decomposition levels with relevant information.

The smoothing effect of this algorithm is clearly evident as the signal is decomposed. On one hand, in the first levels, noise spikes are removed and the waveform is preserved. On the other hand, the last levels do not show the main waveform, but the frequency presented in the envelope of level 5 is the fundamental frequency of the original signal. The level 6 of decomposition has no longer relevant information.

\subsection{Error evaluation}

The described algorithm was applied to five different data sets. For every test, data were acquired in the test setup and, between the data acquisition, the probe was withdrawn from the forward-facing of the membrane and re-aligned. Such variations of the initial conditions permit the understanding of the influence of the probe's positioning and alignment in the final results. The data sets are composed of the generated original signal, the self-mixing signal and time correspondence. All signals were sampled at $100 \mathrm{kHz}$.

After the signal processing, a few quality parameters were extracted by comparing the reconstructed and the original data in order to evaluate the performance of the presented algorithm. Since temporal coherence is the objective of this work, the temporal occurrence of the SP and the reflected wave were previously determined for all data sets. Each data set has ten SP and ten reflected waves because the synthetic pulse waveform period is $1 \mathrm{~s}$. Besides this, the corresponding SP and reflected wave occurrence time were computed in the reconstructed signal using a semi-automatic method. For each peak, the time lags were 

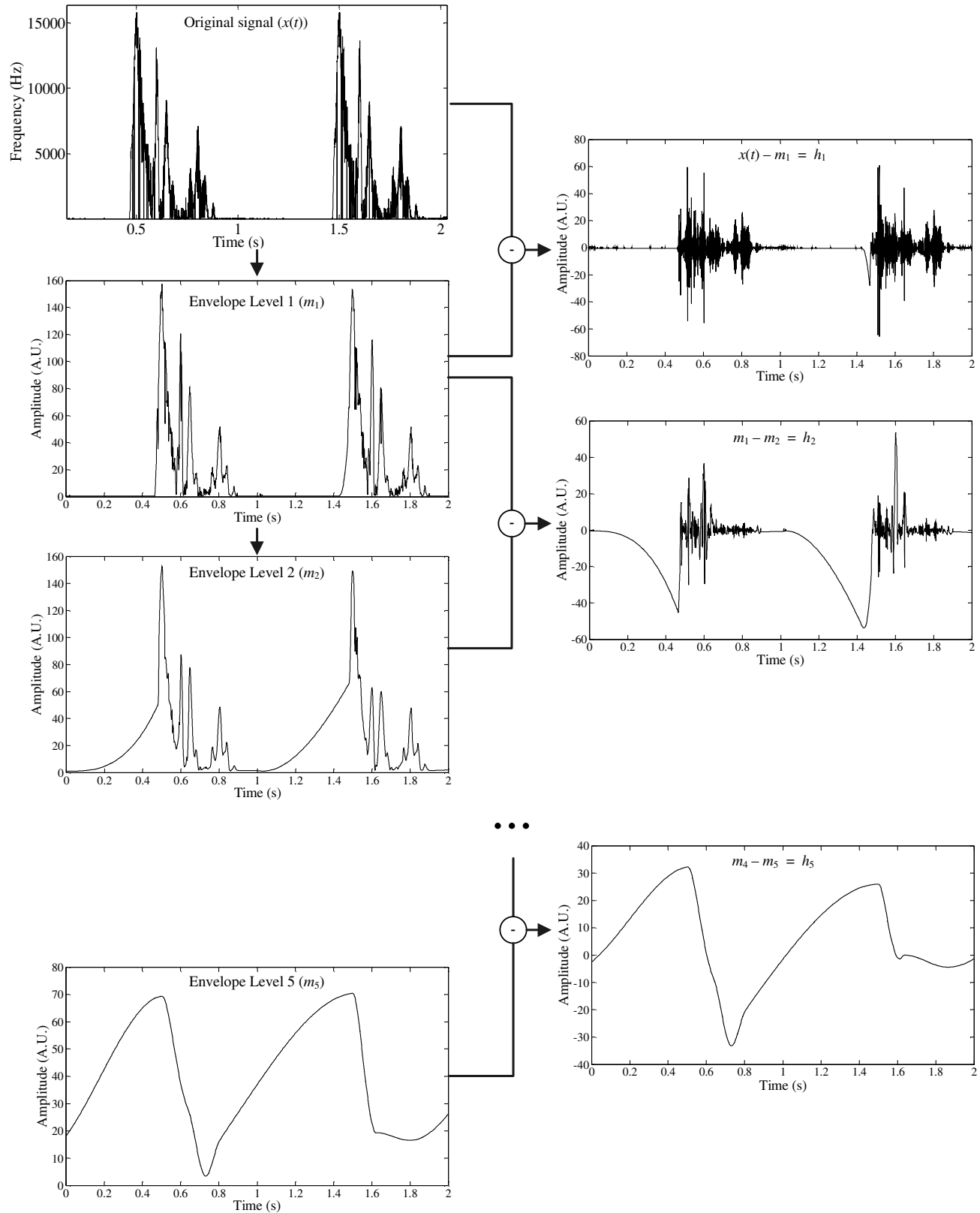

Figure 5. EMD first, second and fifth levels. The envelope and the residue result for each level.

calculated by subtracting the original time occurrence from the reconstructed time occurrence. Finally, the RMS errors for the SP and reflected wave were calculated for each data set. For every data set, the first and second levels of decomposition were considered. The first decomposition level achieves the best performance in all cases and the errors were computed for this decomposition level. 


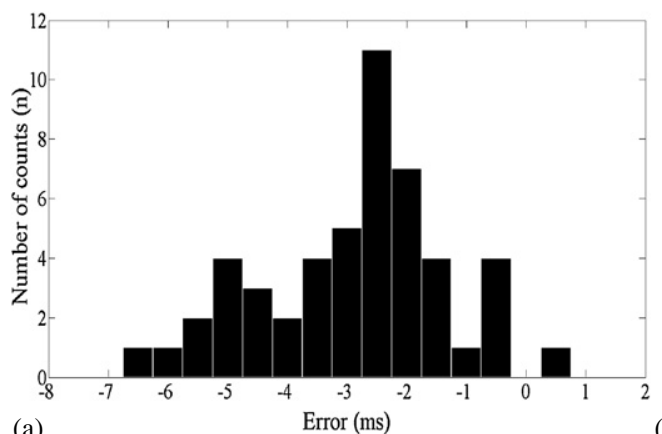

(a)

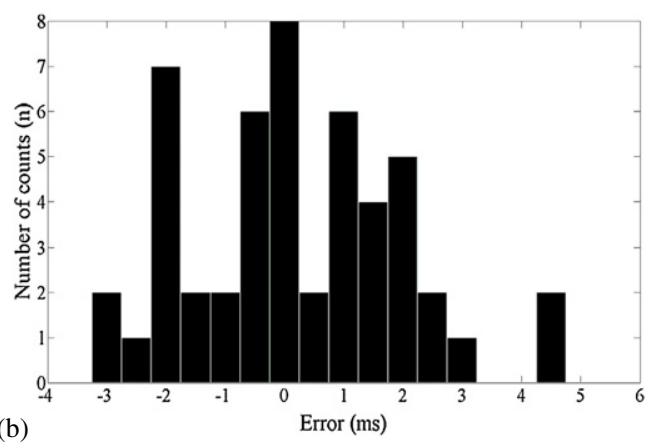

Figure 6. Error dispersion plots for each temporal feature determined by the algorithm. (a) Error dispersion for the SP determination. (b) Error dispersion for the reflected wave.

Table 1. RMS errors between the turning points from the waveform generator and the temporal features determined by the EMD in the first level.

\begin{tabular}{llllll}
\hline & 1st data set & 2nd data set & 3rd data set & 4th data set & 5th data set \\
\hline Systolic peak $e_{\mathrm{RMS}}(\mathrm{ms})$ & 2.5 & 2.7 & 2.9 & 4.7 & 2.9 \\
Reflected wave $e_{\mathrm{RMS}}(\mathrm{ms})$ & 1.4 & 1.3 & 1.9 & 2.1 & 3.0 \\
\hline
\end{tabular}

In terms of RMS errors (table 1), it is important to note the high precision in the point time determination of the reflected wave. For this event, the maximum mean error was $3 \mathrm{~ms}$ for the fifth data set. Between data, small differences in errors occurred. This fact is understandable because the probe alignment influences the quality of the extracted signal but, despite this, the reconstructed algorithm showed high robustness.

With regard to the SP, the RMS errors were slightly larger in almost all sets and never exceeded $3 \mathrm{~ms}$. The high RMS error in their fourth data set is an outlier probably originated by an under optimization of the alignment in the test setup. Lastly, all time errors were small if one considers that under physiological conditions the pulse waveform has a period between 1 and $0.5 \mathrm{~s}$, which is equivalent to 60 and 120 beats per minute.

Data sets were composed of ten SP and ten reflected waves that made a total of 50 feature points for each data set. To get information about the dispersion of the time errors, a histogram (figure 6) was constructed with the time errors of all analyzed data, separated by the feature points (SP and reflected wave).

Considering the histogram of the single errors, a strong conclusion needs to be made. At the time of the determination of the SP, almost all errors had a negative signal. This means that all points were identified with a small delay. This conclusion is acceptable because the spectrograms do not permit to split the original signal into an infinite number of windows. Another cause for this delay is the strong dc component present in the original signal. The need to remove this signal component causes some information loss. Nevertheless, in the reflected wave point histogram, a more balanced distribution of the errors is present. This fact happens because the reflected wave has two rapid turning points (local maximum and local minimum) and the reconstruction algorithm, in some cases, can only identify one of them. Sometimes, the first turning point is identified and other times the second turning point is identified. 

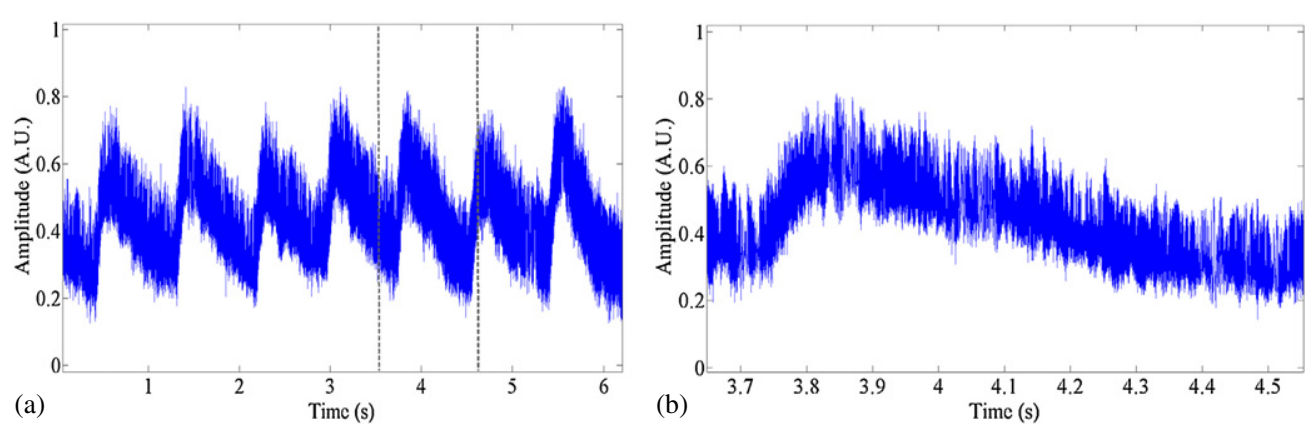

Figure 7. In vivo carotid measurements. (a) Set of cyclic self-mixed laser Doppler signals from the movement of the carotid artery. (b) Detail of the pulse detected by the optical probe.

\section{In vivo carotid measurements}

The expected follow-up of this work started with the acquisition of pulse data in humans. All the measurements were made in the carotid artery, which is the natural probing spot for a waveform measurement, due to the heart proximity and ease of access (i.e. it is close to the skin surface), making easier the measurement itself.

The study protocol was approved by the ethical committee of the Centro Hospitalar e Universitário de Coimbra, EPE Portugal. The subjects were volunteers and gave written informed consent. Measurements were carried out after a rest period in a temperaturecontrolled environment. Each exam procedure consisted in the acquisition of a set of cardiac cycles at the carotid artery for a few minutes, with the patient lying in supine position.

Figure 7 shows a preliminary acquisition in humans using the optical probe. Data were collected directly into a portable computer for a few minutes. The signals show great consistency in the waveform of the pressure wave (figure 7(a)). The self-mixing effect is clearly visible in the Doppler signal (figure 7(b)).

The one-dimensional signal reconstructed from the spectrogram of the set of acquired pulses is shown in figure 8. The reconstructed signal shows the periodic structure of the wave, proportional to the velocity of the distension of the carotid wall.

\section{Conclusions}

In this paper, a new type of optical probe for the measurement of the PPW in a single location and an algorithm for the determination of the temporal specific features of the wave were described. The developed probe has a compact size, is ergonomic, user-friendly and noninvasive, with a truly non-contact operation capability. All the optical system is portable, lightweight and it was designed with low power consumption and low cost materials, in order to be considered as an interesting solution for commercial purposes. The used LD is extensively used in optical drivers, and all the system is based on simple electronic components with a simple optical scheme of one optical axis, for a low cost system. The part of the system with the highest power consumption is the dc-dc converter $(200 \mathrm{~mW})$ making this system appropriate for USB power. The most important characteristics of this system are its high portability, the use of a USB connection for power supply and data transfer, good time response, measurements without complex patient preparation and the absence of complex optical constituents. 


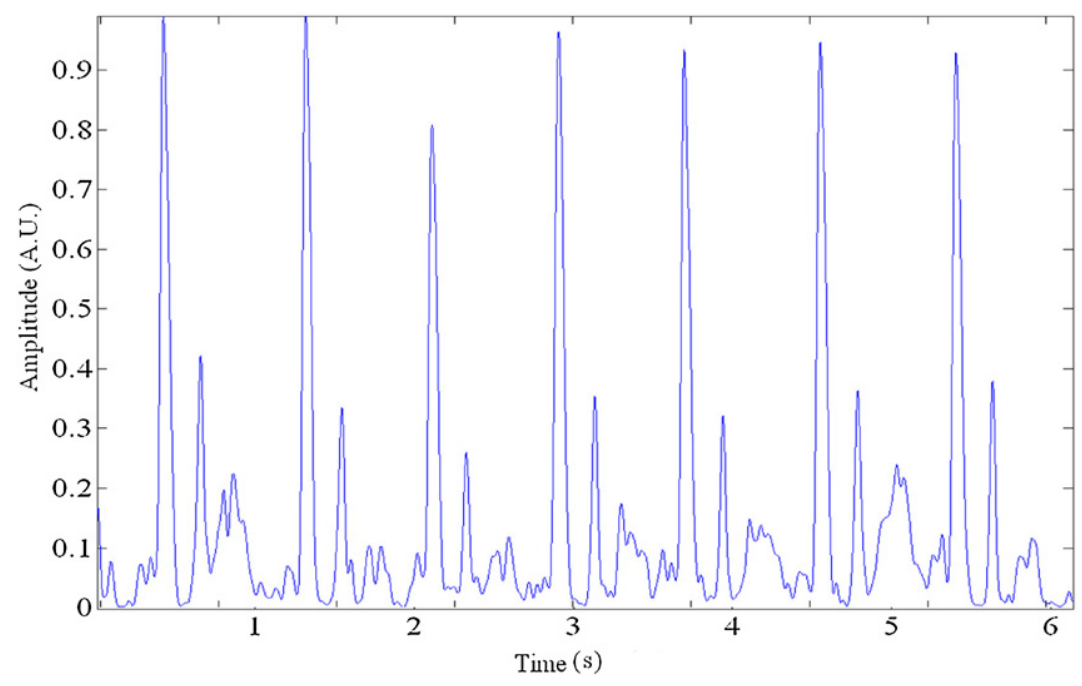

Figure 8. Reconstructed signal from the STFT and EMD.

For the probe's validation, a test setup system was designed, developed and characterized. This test is capable of controlled generation of cardiac pressure waveforms, and well suited to validate feature-extracting algorithms for hemodynamic studies. The developed optical probe has demonstrated good performance in the test setup system.

In addition, the developed algorithm uses well established methods for signal processing of self-mixing Doppler signals, such as the STFT and EMD, to remove noise and apply semi-automatic peak detection. The decomposition levels of the EMD permit the extraction of different pieces of information. The first level allows an easy detection of the feature points in contrast to the fourth/fifth levels that clearly show the basic oscillation frequency. The algorithm is computationally fast and can be implemented on a normal laptop as required for portability. The developed instrumentation and the reconstruction algorithm allow the identification of the pulse waveform feature points with an accuracy of a few milliseconds and high performance in the identification of the reflected wave. The error histograms also showed the robustness of the system because of the restricted distribution of the errors. In comparison to commercial systems, based on tonometry or ultrasound, optic sensors have a small response time and a more rigorous signal is acquired.

The test setup presented is a simple method to simulate the skin behavior in the physiological context. The use of silicone layers instead of a reflector object tries to reproduce a diffuse reflection similar to the one that occurs at the skin, yielding an increase in light scattering. Moreover, the membrane is not completely flat; it has some surface vibrations, which complicate the reflection of light directly back to the optical cavity. Due to these test setup limitations, the optical probe alignment is extremely important in the final signal quality and SNR. In the in vivo carotid measurements, the operator hand vibration needs to be minimized because the laser is a point-like sensor and the PPW needs to be recorded precisely above the carotid site.

Some limitations on the power of the optical probe are imposed by the security rules of laser exposure. Light output power cannot exceed values for which there is risk of damage of organic tissues, such as skin. The high power for the LD improves the SNR of the acquired signals; however, the medical devices have security limitations. 
The use of several data sets, by changing the alignment between the probe and the silicone membrane, are evidence of the robustness of the system. As the developed probe uses an optical signal, it is relevant to note that tests were performed to assess if the room illumination conditions influence the self-mixing signal. No changes in the signal were observed between the dark and illuminated rooms. Pulse waveform reconstruction is a future step on this work through the development of new algorithms. More setup test and in vivo tests are required to fully describe the capabilities of the designed optical system, but the first trials in carotid measurements are promising.

To conclude, the non-invasive and non-contact method proposed by the optical solutions, as well as the implementation of rapid algorithms to assess hemodynamic parameters, will permit a continuous monitoring of the cardiovascular system and give an earlier and accurate diagnosis.

\section{Acknowledgments}

The authors acknowledge the support from Fundação para a Ciência e Tecnologia (FCT) for funding (PTDC/SAU-BEB/100650/2008). The project was developed under the initiative of QREN, funding by UE/FEDER, through COMPETE-Programa Operacional Factores de Competitividade.

\section{References}

Andrade A O, Nasuto S, Kyberd P, Sweeney-Reed C M and Van Kanijn F R 2006 EMG signal filtering based on empirical mode decomposition Biomed. Signal Process. Control $144-55$

Avolio A P, Butlin M and Walsh A 2010 Arterial blood pressure measurement and pulse wave analysis-their role in enhancing cardiovascular assessment Physiol. Meas. 31 R1-47

Boutouyrie P et al 1995 Common carotid artery stiffness and patterns of left ventricular hypertrophy in hypertensive patients Hypertension 25 651-9

Campo A, Segers P and Dirck J 2011 Laser Doppler vibrometry for in vivo assessment of arterial stiffness Proc. IEEE Int. Workshop on Medical Measurements and Applications pp 119-21

Dart A M and Kingwell B A 2001 Pulse pressure-a review of mechanisms and clinical relevance J. Am. Coll. Cardiol. 37 975-84

Figuerias E M 2012 Métodos de instrumentação para fluxometria laser: aplicações à microcirculação sanguínea University of Coimbra

Gallego-Jutglà E and Solé-Casals J 2012 Exploring mEMD for face recognition Proc. 5th Int. Joint Conf. on Biomedical Engineering Systems and Technologies pp 498-503

Giuliani G, Norgia M, Donati S and Bosch T 2002 Laser diode self-mixing technique for sensing applications J. Opt. A: Pure Appl. Opt. 4 S283-94

Guo D and Wang M 2006 Self-mixing interferometer based on temporal-carrier phase-shifting technique for microdisplacement reconstruction Opt. Commun. 263 91-7

Guo D and Wang M 2007 New absolute distance measurement technique with a self-mixing interferometer J. Phys.: Conf. Ser. 48 1381-6

Hai Y, Wei C and Hong Z 2008 TVAR time-frequency analysis for non-stationary vibration signals of spacecraft Chin. J. Aeronaut. 21 423-32

Hast J, Myllyla R, Sorvoja H and Miettinen J 2002 Arterial pulse shape measurement using self-mixing effect in a diode laser Quantum Electron. 32 975-80

Hong H D and Fox MD 1994 No touch pulse measurement by optical interferometry IEEE Trans. Biomed. Eng. 41 1096-9

ICNIRP 2000 Revision of guidelines on limits of exposure to laser radiation of wavelengths between $400 \mathrm{~nm}$ and $1.4 \mu \mathrm{m}$ Health Physics 79 431-40 (available at http://journals.lww.com/health-physics/toc/2000/10000)

Junsheng C, Dejie Y and Yu Y 2006 Research on the intrinsic mode function (IMF) criterion in EMD method Mech. Syst. Signal Process. 20 817-24

Klocke R, Cockcroft J R, Taylor G J, Hall I R and Blake D R 2003 Arterial stiffness and central blood pressure, as determined by pulse wave analysis, in rheumatoid arthritis Ann. Rheum. Dis. 62 414-8 
Korpas D, Hálek J and Dolezal L 2009 Parameters describing the pulse wave Physiol. Res. 58 473-9

Meigas K, Hinrikus H, Lass J and Kattai R 1998 Pulse profile registration using self-mixing in a diode laser IEEE 20th Annu. Int. Conf. of Eng. Med. Biol. Soc. vol 4 pp 1875-8

Meigas K, Kattai R and Lass J 2001 Continuous blood pressure monitoring using pulse wave delay 23rd Ann. Int. Conf. of IEEE Eng. in Med. and Biol. Soc. pp 3171-4

Mya M, Kyu T, Aung Z M and Naing Z M 2009 Design and implementation of active filter for data acquisition system Proc. 2009 Int. Conf. on Information Management and Engineering pp 406-10

Nelson M R, Stepanek J, Cevette M, Covalciuc M, Hurst R T and Tajik A J 2010 Noninvasive measurement of central vascular pressures with arterial tonometry: clinical revival of the pulse pressure waveform? Mayo Clin. Proc. 85 460-72

Pereira T et al 2011 Signal analysis in a new optical pulse waveform profiler for cardiovascular applications Signal and Image Processing and Applications/716: Artificial Intelligence and Soft Computing (SIPA 2011) (Crete, Greece, 22-24 June 2011) pp 19-25

Phillips C, Hedner J, Berend N and Grunstein R 2005 Diurnal and obstructive sleep apnea influences on arterial stiffness and central blood pressure in men Sleep 28 604-9

Qing C 2010 Empirical mode decomposition-based facial pose estimation inside video sequences Opt. Eng. 49037401

Scalise L and Morbiducci U 2008 Non-contact cardiac monitoring from carotid artery using optical vibrocardiography Med. Eng. Phys. 30 490-7

Scalise L and Paone N 2002 Laser Doppler vibrometry based on self-mixing effect Opt. Lasers Eng. 38 173-84

Selzer R H, Mack W J, Lee P L, Kwong-Fu H and Hodis H N 2001 Improved common carotid elasticity and intima-media thickness measurements from computer analysis of sequential ultrasound frames Atherosclerosis 154 185-93

van Ittersum F J et al 2004 Autonomic nervous function, arterial stiffness and blood pressure in patients with Type I diabetes mellitus and normal urinary albumin excretion J. Hum. Hypertens. 18 761-8

Wang M 2001 Fourier transform method for self-mixing interference signal analysis Opt. Laser Technol. 33 409-16

Wang M 2004 Self-mixing microscopic interferometer for the measurement of microprofile Opt. Commun. 238 237-44

Weber T et al 2004 Arterial stiffness, wave reflections, and the risk of coronary artery disease Circulation 109 184-9 